\title{
Association between BrainAgeR scores and Alzhemier's disease brain and plasma biomarkers
}

\author{
Authors: Yousaf Abughofah², Shannon L. Risacher ${ }^{1}$
}

Affiliations: Center for Neuroimaging, Department of Radiology and Imaging Sciences, Indiana Alzheimer's Disease Research Center ${ }^{1}$, Department of Neurology, Indiana University School of Medicine $^{2}$, Indianapolis, Indiana, USA

Background: The goal of this project was to study the association between the age scores assigned by the BrainAgeR computer algorithm and AD biomarker levels in the blood and brain.

Methods: 123 individuals from the Indiana Memory and Aging Study underwent amyloid ( $\left[{ }^{18} \mathrm{~F}\right]$ florbetapir or $\left[{ }^{18} \mathrm{~F}\right]$ florbetaben) and Tau $\left[{ }^{18} \mathrm{~F}\right]$ flortaucipir PET. Another set of 156 individuals from the Indiana Memory and Aging Study underwent plasma testing for Amyloid B 40/42, Tau and neurofilament light chain (NFL). Additionally, all participants underwent structural MRI and were processed using BrainAgeR to receive a "brain age" score. Partial Person correlation models were used to evaluate the relationship between BrainAge difference scores (Chronological age-BrainAge) and levels of Amyloid and Tau in the brain and plasma. Age and diagnosis were evaluated as covariates but did not change the observed pattern of results.

Results: Significant negative association between BrainAge difference scores and Tau uptake was observed across the neuroimaging group. This correlation persisted when analysis was limited to MCI/AD subjects but was lost when analysis was only limited to CN/SCD subjects. Across all participants in the neuroimaging group, significant negative associations were found between BrainAge differences and the levels of Amyloid deposition in the global cortex. Significant positive association was found between AB42/40 ratio and BrainAge difference scores across the entire plasma group. Significant negative relationships found between NFL and AB40 and BrainAge difference scores when analyzed in the CN/SCD group, but no statistically significant relationship was found when only the MCI/AD group was analyzed.

Conclusion: BrainAge difference scores had a statistically significant association with various biomarkers of $A D$ depending on the level of diagnosis, with cognitively normal and less impaired subjects showing an association with plasma amyloid and more impaired subjects showing an association with Tau deposition in the brain. Future studies in larger samples are warranted. 